\title{
Optimal copying of entangled two-qubit states
}

\author{
J. Novotný( ${ }^{(1)}$, G. Alber ${ }^{(2)}$, I. Jex ${ }^{(1)}$ \\ (1) Department of Physics, FJFI ČVUT, Břehová 7, 11519 Praha 1 - Staré Město, Czech Republic \\ (2) Institut für Angewandte Physik, Technische Universität Darmstadt, D-64289 Darmstadt, Germany
}

(Dated: today)

\begin{abstract}
We investigate the problem of copying pure two-qubit states of a given degree of entanglement in an optimal way. Completely positive covariant quantum operations are constructed which maximize the fidelity of the output states with respect to two separable copies. These optimal copying processes hint at the intricate relationship between fundamental laws of quantum theory and entanglement.
\end{abstract}

PACS numbers: 03.67.Mn,03.65.Ud

\section{INTRODUCTION}

The optimal copying (cloning) of quantum states is an elementary process of central interest in quantum information processing 1]. As arbitrary quantum states cannot be copied perfectly, the interesting question arises as to which extent a quantum process can perform this task in an optimal way. Recently, much work has been devoted to the copying of pure quantum states $[2,3,4,5,6,7,8,9,10]$. These investigations demonstrate that the maximal fidelity which can be achieved in an optimal copying process depends on characteristic properties of the set of states which are to be copied.

Motivated by the important role entanglement is playing in the area of quantum information processing in this paper we address the question of copying pure entangled two-qubit states in an optimal way. Though much work has already been devoted to the copying of pure single particle states, first investigations addressing the problem of copying entanglement have been performed only recently [10]. In this latter work it was demonstrated that entanglement cannot be copied perfectly. Thus, if one can find a quantum operation which perfectly duplicates entanglement of all maximally entangled qubit pairs, it necessarily cannot respect separability of the two identical copies produced. Furthermore, for the special case of maximally entangled two-qubit states first copying processes were constructed which maximize the fidelity of each two-qubit copy separately.

In this paper we address the general problem of copying pure two-qubit states of an arbitrarily given degree of entanglement in an optimal way. In particular, we are interested in constructing completely positive quantum operations which do not only copy the pure entangled input state but which also guarantee separability of the resulting two-qubit copies in an optimal way. Our motivation for restricting our investigation to two-qubit input states is twofold. Firstly, qubit states still play a dominant role in the area of quantum information processing. Secondly, it is expected that in this simplest case the intricate relationship between entanglement and limits imposed on quantum copying processes by the fundamental laws of quantum theory are exposed in a particularly transparent way.

This paper is organized as follows: In Sec. II we briefly recapitulate the basic relation between optimal copying processes and corresponding covariant quantum processes which maximize the fidelity of the output states with respect to separable copies. In Sec. III the most general covariant quantum processes are constructed which are consistent with the linear character of quantum maps and which copy arbitrary pure two-qubit quantum states of a given degree of entanglement. In Sec. IV the additional constraints are investigated which result from the positivity of these quantum maps. Based on these results the parameters of the optimal copying processes are determined. In Sec. $\mathrm{V}$ it is shown that these optimal covariant quantum maps can be realized by completely positive deterministic quantum operations. Thus, they may be implemented by an appropriate unitary transformation and a subsequent measurement process involving additional ancilla qubits. Basic physical properties of the resulting optimally copied states are discussed in Sec.VI.

\section{OPTIMAL COPYING OF ENTANGLED TWO-QUBIT STATES}

In this section basic connections between optimal quantum mechanical copying processes and covariant quantum processes are summarized and specialized to the problem of copying pure bipartite entangled two-qubit states in an optimal way.

In order to put the problem into perspective let us consider two distinguishable spin-1/2 particles (qubits). Their associated four dimensional Hilbert space $\mathcal{H}$ can be decomposed into classes of pure two-qubit states $\Omega_{\alpha}$ of a given degree of entanglement $\alpha$ (the parameter $\alpha$ determines the amount of entanglement in the bipartite system described by states from $\Omega_{\alpha}$ ). These classes are represented by the sets 


$$
\Omega_{\alpha}=\left\{\left(U_{1} \otimes U_{2}\right)\left(\alpha|\uparrow\rangle_{1} \otimes|\uparrow\rangle_{2}+\sqrt{1-\alpha^{2}}|\downarrow\rangle_{1} \otimes|\downarrow\rangle_{2}\right) \mid U_{1}, U_{2} \in S U(2)\right\} .
$$

Thereby the parameter $\alpha(0 \leq \alpha \leq 1)$ characterizes the degree of entanglement of the pure states in a given class $\Omega_{\alpha}$ and the kets $|\uparrow\rangle$ and $|\downarrow\rangle$ constitute an orthonormal basis of the two-dimensional single-qubit Hilbert spaces of each of the qubits (distinguished by the subscripts 1 and 2). Relation (11) takes into account that local unitary operations of the form $U_{1} \otimes U_{2}$ are the most general transformations which leave the degree (measure) of entanglement $\alpha$ of a bipartite quantum state invariant. Due to the symmetry relation $\Omega_{\alpha}=\Omega_{\sqrt{1-\alpha^{2}}}$ we can restrict our subsequent discussion to the parameter range $0 \leq \alpha \leq 1 / \sqrt{2}$. Note that in the special case $\alpha=0$ the two-qubit state is separable whereas in the opposite extreme case $\alpha=1 / \sqrt{2}$ it is maximally entangled. Furthermore, it should be noted that each class $\Omega_{\alpha}$ contains an orthonormal basis of $\mathcal{H}$.

We are interested in constructing quantum processes $T_{\alpha}$ which copy an arbitrary pure two-qubit state, say $|\psi\rangle \in \Omega_{\alpha}$, in an optimal way, i.e.

$$
T_{\alpha}: \rho_{0} \equiv \rho_{\text {in }} \otimes \rho_{\text {ref }} \longrightarrow \rho_{\text {out }},
$$

with $\rho_{i n}=|\psi\rangle\langle\psi|$ denoting the density operator of the the input state. The resulting four-qubit output state is denoted by $\rho_{\text {out }}$. The appropriately chosen two-qubit quantum state $\rho_{\text {ref }}$ characterizes the initial state of the copying device which is independent of the input state. According to the fundamental laws of quantum theory the quantum map $T_{\alpha}$ has to be linear and completely positive [11, 12, 13].

The fidelity $F=\left\langle\psi\left|\otimes\left\langle\psi\left|\rho_{\text {out }}\right| \psi\right\rangle \otimes\right| \psi\right\rangle$ constitutes a convenient quantitative measure of how close an output state $\rho_{\text {out }}$ resembles two ideal separable copies of the original input state $\rho_{\text {in }}=|\psi\rangle\langle\psi|$. Consequently, the smallest achievable fidelity, i.e.

$$
\mathcal{L}\left(T_{\alpha}\right)=\inf _{|\psi\rangle \Omega_{\alpha}}\left\langle\psi\left|\otimes\left\langle\psi\left|\rho_{\text {out }}\right| \psi\right\rangle \otimes\right| \psi\right\rangle,
$$

characterizes the quality of a copying process for a given class of entangled input states $|\psi\rangle \in \Omega_{\alpha}$. Thus, constructing an optimal copying process is equivalent to maximizing $\mathcal{L}\left(T_{\alpha}\right)$ over all possible quantum processes. Let us denote this optimal fidelity by $\widehat{\mathcal{L}_{\alpha}} \equiv \sup _{T_{\alpha}} \mathcal{L}\left(T_{\alpha}\right)$. It has been shown [3, 4] that for any optimal quantum copying process $\widehat{T_{\alpha}}$ one can always find an equivalent covariant quantum process with the characteristic property

$$
\rho_{\text {out }}\left[U_{1} \otimes U_{2} \rho_{\text {in }} U_{1}^{\dagger} \otimes U_{2}^{\dagger}\right]=\mathcal{U} \rho_{\text {out }}\left(\rho_{\text {in }}\right) \mathcal{U}^{\dagger}
$$

with $\mathcal{U}=U_{1} \otimes U_{2} \otimes U_{1} \otimes U_{2}$. Thus, this equivalent covariant quantum process yields the same optimal fi- delity $\widehat{\mathcal{L}_{\alpha}}$ for all possible two-qubit input states $|\psi\rangle \in \Omega_{\alpha}$. Thereby, $U_{1}, U_{2} \in S U_{2}$ are arbitrary unitary one-qubit transformations. A proof of this theorem is sketched in Appendix A. This observation allows us to restrict our further search for optimal copying processes of entangled pure two-qubit states to covariant quantum processes which maximize the fidelity of Eq.(3).

At this point we want to emphasize that in this work we are concerned with optimal entanglement processes which maximize the fidelity of Eq.(3). Thus, the covariant copying processes we are looking for are designed for producing output states of the form $|\psi\rangle \otimes|\psi\rangle$, i.e. two separable pairs of pure entangled two-qubit states, with the highest possible probability for all possible two-qubit input states $|\psi\rangle \in \Omega_{\alpha}$. As we are focusing on separable copies of the input states these processes do not necessarily also maximize the fidelity $F^{\prime}$ of the output state with respect to each copy separately, i.e. with respect to $F^{\prime}=\operatorname{Tr}\left\{|\psi\rangle\langle\psi| \otimes \mathbf{1} \rho_{\text {out }}(|\psi\rangle\langle\psi|)\right\}$. These latter processes were studied in Ref. [10], for example, for the special case of maximally entangled pure input states, i.e. $\alpha=1 / \sqrt{2}$.

\section{COVARIANT LINEAR QUANTUM PROCESSES}

In this section all possible covariant copying processes are constructed which are consistent with the linear character of general quantum maps of the form of Eq.(2).

In view of the covariance condition (44) all possible quantum maps of the form (2) can be characterized by the output states $\rho_{\text {out }}\left(\rho_{\text {in }}\right)$ which originate from one arbitrarily chosen pure input state, say $|\psi\rangle=\alpha|\uparrow\rangle_{1} \otimes \mid \uparrow$ \rangle$_{2}+\sqrt{1-\alpha^{2}}|\downarrow\rangle_{1} \otimes|\downarrow\rangle_{2}$ with $0 \leq \alpha \leq 1 / \sqrt{2}$. In order to fulfill Eq.(4) the two-qubit reference state $\rho_{\text {ref }}$ of Eq.(2) has to be invariant under arbitrary local unitary transformations of the form $U_{1} \otimes U_{2}$. Therefore, the initial state of the covariant quantum map is of the form [4]

$$
\rho_{0}=\rho_{\text {in }} \otimes \frac{1}{4} \mathbf{1} \equiv|\psi\rangle\langle\psi| \otimes \frac{1}{4} \mathbf{1} .
$$

In order to implement the covariance condition of Eq.(4) it is convenient to decompose this quantum state into irreducible two-qubit tensor operators $T^{(1,3)}\left(J^{\prime}, J\right)_{K Q}$ and $T^{(2,4)}\left(J^{\prime}, J\right)_{K Q}$ [14, 15] with respect to qubits one and three on the one hand and qubits two and four on the other hand. Performing an arbitrary unitary transformation of the form $U_{1} \otimes U_{2} \otimes U_{1} \otimes U_{2}$ with $U_{1}, U_{2} \in S U_{2}$, for example, a product of such tensor operators transforms according to 


$$
\mathcal{U} T^{(1,3)}\left(J_{1}^{\prime} J_{1}\right)_{K_{1} Q_{1}} \otimes T^{(2,4)}\left(J_{2}^{\prime} J_{2}\right)_{K_{2} Q_{2}} \mathcal{U}^{\dagger}=\sum_{q_{1}, q_{2}} D\left(U_{1}\right)_{q_{1} Q_{1}}^{\left(K_{1}\right)} D\left(U_{2}\right)_{q_{2} Q_{2}}^{\left(K_{2}\right)} T^{(1,3)}\left(J_{1}^{\prime} J_{1}\right)_{K_{1} q_{1}} \otimes T^{(2,4)}\left(J_{2}^{\prime} J_{2}\right)_{K_{2} q_{2}}
$$

with $\mathcal{U}=U_{1} \otimes U_{2} \otimes U_{1} \otimes U_{2}$. Thereby, $D\left(U_{j}\right)(j=1,2)$ denote the relevant rotation operators and $D\left(U_{j}\right)_{q_{j} Q_{j}}^{\left(K_{j}\right)}$ are their associated rotation matrices [15]. The quantum numbers $J_{j}, J_{j}^{\prime}$ denote the total angular momenta of the relevant two-qubit quantum states and the parameters $K_{j}$ indicate the irreducible subspaces of the relevant representations. For the sake of convenience some basic relations of these irreducible two-qubit tensor opera- tors are summarized in Appendix B. It is apparent from Eq.(6) that an arbitrary unitary transformation of the form $U_{1} \otimes U_{2} \otimes U_{1} \otimes U_{2}$ with $U_{1}, U_{2} \in S U_{2}$ mixes the parameters $q_{1}$ and $q_{2}$ within each irreducible representation separately. In terms of these irreducible tensor operators an arbitrary initial state $\rho_{0}$ of the form of Eq.(5) can be decomposed according to (compare with Eq.(B4)

$$
\rho_{0}=\sum_{j_{1}, \ldots, j_{4}, K, Q, K^{\prime}, Q^{\prime}} T^{(1,3)}\left(j_{1}, j_{3}\right)_{K Q} \otimes T^{(2,4)}\left(j_{2}, j_{4}\right)_{K^{\prime} Q^{\prime}}\left\langle T^{(1,3) \dagger}\left(j_{1}, j_{3}\right)_{K Q} T^{(2,4) \dagger}\left(j_{2}, j_{4}\right)_{K^{\prime} Q^{\prime}}\right\rangle
$$

with the expansion coefficients

$$
\left\langle T^{(1,3) \dagger}\left(j_{1}, j_{3}\right)_{K Q} T^{(2,4) \dagger}\left(j_{2}, j_{4}\right)_{K^{\prime} Q^{\prime}}\right\rangle \equiv \operatorname{Tr}\left\{\left(T^{(1,3) \dagger}\left(j_{1}, j_{3}\right)_{K Q} \otimes T^{(2,4) \dagger}\left(j_{2}, j_{4}\right)_{K^{\prime} Q^{\prime}}\right) \rho_{0}\right\} .
$$

Thereby, $\operatorname{Tr}$ denotes the trace over the four-qubit Hilbert space of the system- and device qubits. In view of the basic transformation property of Eq.(6) the most general output state resulting from a linear and covariant quantum map is given by

$\rho_{\text {out }}\left(\rho_{\text {in }}\right)=\sum_{j_{1}, \ldots, j_{4}, K, Q, K^{\prime}, Q^{\prime}} \alpha\left(j_{1}, j_{3}, j_{2}, j_{4}\right)_{K K^{\prime}} T^{(1,3)}\left(j_{1}, j_{3}\right)_{K Q} T^{(2,4)}\left(j_{2}, j_{4}\right)_{K^{\prime} Q^{\prime}}\left\langle T^{(1,3) \dagger}\left(j_{1}, j_{3}\right)_{K Q} T^{(2,4) \dagger}\left(j_{2}, j_{4}\right)_{K^{\prime} Q^{\prime}}\right\rangle$.

According to the fundamental laws of quantum theory the unknown coefficients $\alpha\left(j_{1}, j_{3}, j_{2}, j_{4}\right)_{K K^{\prime}}$ are necessarily restricted by the fact that $\rho_{\text {out }}$ has to be a nonnegative operator. In particular, being a Hermitian operator the output state $\rho_{\text {out }}$ has to fulfill the relations

$$
\alpha\left(j_{1}, j_{3}, j_{2}, j_{4}\right)_{K K^{\prime}}=\alpha\left(j_{3}, j_{1}, j_{4}, j_{2}\right)_{K K^{\prime}}^{*}
$$

Further restrictions on these unknown coefficients are obtained from the explicit form of the input state $\rho_{0}$, i.e.

$$
\rho_{0}=\frac{|\alpha|^{2}}{4}\left\{\frac{1}{\sqrt{2}} T^{(1,3)}(1,1)_{10}+\frac{3}{2} T^{(1,3)}(1,1)_{00}+\frac{1}{2} T^{(1,3)}(0,0)_{00}-\frac{1}{2} T^{(1,3)}(0,1)_{1,0}+\frac{1}{2} T^{(1,3)}(1,0)_{10}\right\} \otimes
$$




$$
\begin{aligned}
&\left\{\frac{1}{\sqrt{2}} T^{(2,4)}(1,1)_{10}+\frac{3}{2} T^{(2,4)}(1,1)_{00}+\frac{1}{2} T^{(2,4)}(0,0)_{00}-\frac{1}{2} T^{(2,4)}(0,1)_{1,0}+\frac{1}{2} T^{(2,4)}(1,0)_{10}\right\}+ \\
& \frac{|\beta|^{2}}{4}\left\{\frac{-1}{\sqrt{2}} T^{(1,3)}(1,1)_{10}+\frac{3}{2} T^{(1,3)}(1,1)_{00}+\frac{1}{2} T^{(1,3)}(0,0)_{00}+\frac{1}{2} T^{(1,3)}(0,1)_{1,0}-\frac{1}{2} T^{(1,3)}(1,0)_{10}\right\} \otimes \\
&\left\{\frac{-1}{\sqrt{2}} T^{(2,4)}(1,1)_{10}+\frac{3}{2} T^{(2,4)}(1,1)_{00}+\frac{1}{2} T^{(2,4)}(0,0)_{00}+\frac{1}{2} T^{(2,4)}(0,1)_{1,0}-\frac{1}{2} T^{(2,4)}(1,0)_{10}\right\}+ \\
& \frac{\alpha \beta^{*}}{8}\left\{-\sqrt{2} T^{(1,3)}(1,1)_{11}+T^{(1,3)}(0,1)_{11}-T^{(1,3)}(1,0)_{11}\right\} \otimes \\
&\left\{-\sqrt{2} T^{(2,4)}(1,1)_{11}+T^{(2,4)}(0,1)_{11}-T^{(2,4)}(1,0)_{11}\right\}+ \\
& \frac{\alpha^{*} \beta}{8}\left\{\sqrt{2} T^{(1,3)}(1,1)_{1-1}-T^{(1,3)}(0,1)_{1-1}+T^{(1,3)}(1,0)_{1-1}\right\} \otimes \\
&\left\{\sqrt{2} T^{(2,4)}(1,1)_{1-1}-T^{(2,4)}(0,1)_{1-1}+T^{(2,4)}(1,0)_{1-1}\right\}
\end{aligned}
$$

with $\beta=\sqrt{1-\alpha^{2}}$. Thus, according to Eq.(10) the most general output state of Eq. (8) generally depends on 17 coefficients, namely

$$
\begin{aligned}
& \alpha(1,1,1,1)_{11}=A_{1}, \alpha(1,1,1,1)_{10}=A_{2}, \\
& \alpha(1,1,1,0)_{11}=A_{3}, \alpha(1,1,0,0)_{10}=A_{4}, \\
& \alpha(1,1,1,1)_{01}=A_{5}, \alpha(1,1,1,1)_{00}=A_{6}, \\
& \alpha(1,1,1,0)_{11}=A_{7}, \alpha(1,1,0,0)_{00}=A_{8}, \\
& \alpha(1,0,1,1)_{11}=A_{9}, \alpha(1,0,1,1)_{10}=A_{10}, \\
& \alpha(1,0,1,0)_{11}=A_{11}, \alpha(1,0,0,0)_{10}=A_{12}, \\
& \alpha(0,0,1,1)_{01}=A_{13}, \alpha(0,0,1,1)_{00}=A_{14}, \\
& \alpha(0,0,1,0)_{01}=A_{15}, \alpha(0,0,0,0)_{00}=A_{16}, \\
& \alpha(1,0,0,1)_{11}=A_{17} .
\end{aligned}
$$

These parameters determine all linear covariant quantum processes with a Hermitian output state $\rho_{\text {out }}\left(\rho_{\text {in }}\right)$ provided the coefficients $A_{1}, A_{2}, A_{4}, A_{5}, A_{6}, A_{8}, A_{13}, A_{14}, A_{16}$ are real-valued. The explicit form of the output state $\rho_{\text {out }}\left(\rho_{\text {in }}\right)$ is given in Appendix C (compare with Eq. (C1)). Proper normalization of the output state requires $\operatorname{Tr}\left\{\rho_{\text {out }}\left(\rho_{\text {in }}\right)\right\}=1$ which implies

$$
\frac{1}{16}\left(9 A_{6}+3 A_{8}+3 A_{14}+A_{16}\right)=1
$$

\section{OPTIMAL COVARIANT COPYING PROCESSES}

In this section the special covariant quantum processes are determined which copy pure entangled two-qubit states of a given degree of entanglement $\alpha$ with the highest possible fidelity.
For this purpose we start from the most general output state which is consistent with the linear and covariant character of the copying process as determined by Eqs.(8), (91), (12) and by an arbitrary combination of the possible non-zero parameters $A_{1}, \ldots, A_{17}$ of (11). The non-negativity of this output state on the one hand and the optimization of its fidelity on the other hand impose further restrictions on these parameters.

The non-negativity of the output state implies that the inequality $\left\langle\chi\left|\rho_{\text {out }}\left(\rho_{\text {in }}\right)\right| \chi\right\rangle \geq 0$ has to be fulfilled for arbitrary pure four-qubit states $|\chi\rangle$. As outlined in appendix $\mathrm{C}$ this condition gives rise to the set of inequalities

$$
\begin{aligned}
& A_{6} \geq 0, A_{8} \geq 0, A_{14} \geq 0, A_{16} \geq 0,\left|A_{1}\right| \leq A_{6}, \\
& \left|\left(2 \alpha^{2}-1\right) A_{4}\right| \leq A_{8},\left|\left(2 \alpha^{2}-1\right) A_{13}\right| \leq A_{14}, \\
& \left|\left(2 \alpha^{2}-1\right) A_{2}\right| \leq A_{6},\left|\left(2 \alpha^{2}-1\right) A_{5}\right| \leq A_{6}
\end{aligned}
$$

and

$$
\begin{aligned}
\left|A_{11}\right|^{2} \leq A_{16} A_{6},\left|A_{17}\right|^{2} \leq & A_{14} A_{8}, \\
A_{6}\left|\left(2 \alpha^{2}-1\right)\left(A_{2}+A_{5}\right)\right|^{2} \leq & \left(A_{1}+A_{6}\right)^{2} A_{6}-\quad(14) \\
& 8 \alpha^{2}\left(1-\alpha^{2}\right) A_{1}^{2}\left(A_{1}+A_{6}\right) .
\end{aligned}
$$

In particular, in the special case $A_{1}=A_{6} \neq 0$ the last inequality of (14) implies

$$
\left|\left(2 \alpha^{2}-1\right)\left(A_{2}+A_{5}\right)\right| \leq \sqrt{4 A_{6}^{2}-16 \alpha^{2}\left(1-\alpha^{2}\right) A_{6}^{2}} .
$$

The fidelity $F$ of the output state $\rho_{\text {out }}\left(\rho_{\text {in }}\right)$ of Eq. (8) with respect to the ideal pure two-qubit output state $|\psi\rangle \otimes|\psi\rangle$ with $|\psi\rangle=\alpha|\uparrow\rangle_{1} \otimes|\uparrow\rangle_{2}+\sqrt{1-\alpha^{2}}|\downarrow\rangle_{1} \otimes|\downarrow\rangle_{2}$ is given by

$$
F \equiv\left\langle\psi\left|\otimes\left\langle\psi\left|\rho_{\text {out }}\right| \psi\right\rangle \otimes\right| \psi\right\rangle=\frac{1}{16}\left\{A_{1}\left(1+2 \alpha^{2}\left(1-\alpha^{2}\right)\right)+\left(2 \alpha^{2}-1\right)^{2}\left(A_{2}+A_{5}\right)+A_{6}\left(1-\alpha^{2}\left(1-\alpha^{2}\right)\right)+\alpha^{2}\left(1-\alpha^{2}\right) A_{16}+\right.
$$




$$
\left.6 \alpha^{2}\left(1-\alpha^{2}\right) \operatorname{Re} A_{11}\right\}
$$

Besides the parameter $\alpha$ determining the degree of entanglement of the input state $|\psi\rangle$ this fidelity depends on the six parameters $A_{1}, A_{2}, A_{5}, A_{6}, A_{11}, A_{16}$. An upper bound of this fidelity can be derived with the help of the inequal- ities (14), (15) and with the relation $A_{16} \leq 16-9 A_{6}$ which is obtained from the normalization condition (12), i.e.

$$
F \leq \frac{1}{16}\left\{A_{6}\left(4-16 \alpha^{2}\left(1-\alpha^{2}\right)\right)+16 \alpha^{2}\left(1-\alpha^{2}\right)+6 \alpha^{2}\left(1-\alpha^{2}\right) \sqrt{A_{6}\left(16-9 A_{6}\right)}\right\}
$$

This upper bound is attained provided the conditions $A_{16}=16-9 A_{6}, A_{11}=\sqrt{A_{6}\left(16-9 A_{6}\right)}$ and $A_{1}=A_{6}=$ $\left(A_{2}+A_{5}\right) / 2$ are fulfilled. Maximizing the right hand side of Eq.(17) with respect to the single parameter $A_{6}$ we finally arrive at the inequality

$$
\begin{aligned}
F \leq F_{\max } \equiv & \frac{2}{9}\left(1-4 \alpha^{2}\left(1-\alpha^{2}\right)\right)(1+\sqrt{v})+ \\
& \alpha^{2}\left(1-\alpha^{2}\right)(1+\sqrt{1-v})
\end{aligned}
$$

with

$$
v=1-\frac{81 \alpha^{4}\left(1-\alpha^{2}\right)^{2}}{145 \alpha^{4}\left(1-\alpha^{2}\right)^{2}-32 \alpha^{2}\left(1-\alpha^{2}\right)+4} .
$$

This upper bound of relation (18) is reached provided the parameters of the covariant copying process fulfill the relations

$$
\begin{aligned}
& A_{1}=\frac{A_{2}+A_{5}}{2}=A_{6} \equiv A_{6}^{\max }=\frac{8}{9}(1+\sqrt{v}) \\
& A_{16}=16-9 A_{6}, A_{11}=\sqrt{A_{6}\left(16-9 A_{6}\right)} .
\end{aligned}
$$

Consistent with the inequalities (13), (14) and with Eq. (C2) the remaining parameters of the covariant copying process which do not explicitly determine the fidelity must be chosen in the following way

$$
\begin{aligned}
A_{2}-A_{5}= & A_{4}=A_{3}=A_{7}=A_{8}=A_{9}=A_{10}= \\
& A_{12}=A_{13}=A_{14}=A_{15}=A_{17}=0 .
\end{aligned}
$$

With the help of Eq. (C2) it is straightforward to check that for these parameters the output state $\rho_{\text {out }}\left(\rho_{\text {in }}\right)$ is a non-negative operator.

Thus, consistent with the fundamental laws of quantum theory the output state of a covariant quantum process which copies all pure two-qubit states of the same degree of entanglement $\alpha$ with the maximal fidelity $F_{\max }$ is given by Eq.(8) with the parameters (11) being determined by Eqs. (20) and (21) (compare also with Eq.(C2)).

The fidelity $F_{\max }$ of this optimal covariant copying process and its dependence on the degree of entanglement $\alpha$ of the pure two-qubit input state is depicted in

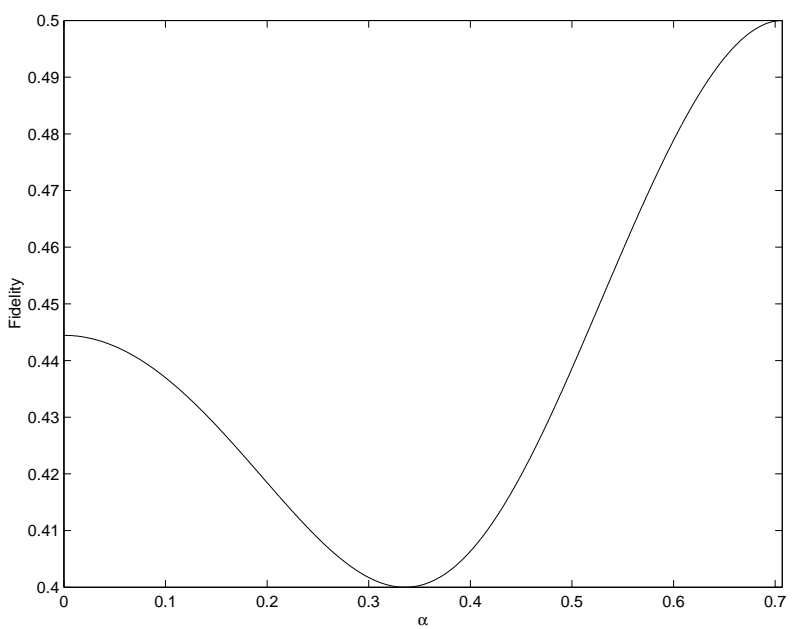

FIG. 1: Fidelity of the optimal covariant copying process and its dependence on the degree of entanglement $\alpha$ of a pure two-qubit input state. The fidelity varies between 0.4 and 0.5 .

Fig 1 This fidelity oscillates between a minimum value of $F=0.4$ which is assumed at $\alpha=\sqrt{1 / 2-\sqrt{15} / 10}$ and a maximum value of $F=1 / 2$ which is assumed at $\alpha=1 / \sqrt{2}$. The value $\alpha=0$ corresponds to the optimal copying of two arbitrary (generally different) separable qubit states. Consistent with known results on optimal cloning of arbitrary single qubit states in this latter case the fidelity $F_{\max }$ assumes the value $F=(2 / 3)^{2}$. From Fig प it is also apparent for which degrees of entanglement $\alpha$ it is easier to copy entangled states than separable ones.

At this point it is worth mentioning major differences between our results and the previously published results on the copying of maximally entangled states of Ref. [10]. In our treatment we are interested in obtaining two separable copies, say $|\psi\rangle \otimes|\psi\rangle$, of entangled two-qubit states $|\psi\rangle \in \Omega_{\alpha}$ of a given degree of entanglement. Correspondingly, we are optimizing 
the two-pair fidelity $F=\left\langle\psi\left|\otimes\left\langle\psi\left|\rho_{\text {out }}\right| \psi\right\rangle \otimes\right| \psi\right\rangle$, for $0 \leq \alpha \leq 1 / \sqrt{2}$. In contrast, in Ref. [10] the optimization of the single-pair fidelities $F_{1}^{\prime}=\operatorname{Tr}\left\{|\psi\rangle\langle\psi| \otimes \mathbf{1}_{34} \rho_{\text {out }}\right\}$ and $F_{2}^{\prime}=\operatorname{Tr}\left\{\mathbf{1}_{12} \otimes|\psi\rangle\langle\psi| \rho_{\text {out }}\right\}$ was investigated for the special case of maximally entangled input states, i.e. $\alpha=1 / \sqrt{2}$. Thus, this latter process does not simultaneously also optimize the separability of the copies. For maximally entangled input states our optimized process yields for these latter figure of merits, for example, the values $F_{1}^{\prime}=F_{2}^{\prime}=0.67$ which is below the optimal value of 0.7171 presented in Ref. [10]. But, for the same value of $\alpha=1 / \sqrt{2}$ the figure of merit of Eq.(16) yields for our optimal copying process the values $F_{\max }=0.5$ whereas the copying process of Ref. [10] yields the smaller value of $F_{\max }=0.458$ because this latter process does not op- timize with respect to two separable copies.

\section{OPTIMAL COVARIANT COPYING PROCESSES AS COMPLETELY POSITIVE QUANTUM OPERATIONS}

In this section it is demonstrated that the covariant optimal copying processes derived in Sec. IV are completely positive deterministic quantum operations. Thus, they can be implemented by unitary quantum transformations with the help of additional ancilla qubits.

Using Eqs. (8), (20), (21) it is straightforward to demonstrate that the output state of the optimal covariant quantum copying process can be written in the form

$$
\rho_{\text {out }}(|\psi\rangle\langle\psi|)=K|\psi\rangle\left\langle\psi\left|\otimes \frac{1}{4} \mathbf{1} K^{\dagger} \equiv \sum_{i, j=0,1} \mathcal{K}_{i j}\right| \psi\right\rangle\langle\psi| \mathcal{K}_{i j}
$$

with the operators

$$
\begin{aligned}
K & =\sqrt{A_{1}} P_{T}^{(1,3)} \otimes P_{T}^{(2,4)}+\sqrt{A_{16}} P_{S}^{(1,3)} \otimes P_{S}^{(2,4)}=K^{\dagger}, \\
\mathcal{K}_{i j} & =\frac{K}{2}|i\rangle_{3} \otimes|j\rangle_{4} .
\end{aligned}
$$

Thereby, $P_{T}^{(a, b)}=\sum_{M=0, \pm 1}|1 M\rangle\langle 1 M|\otimes| 1 M\rangle\langle 1 M|$ and $P_{S}^{(a, b)}=|00\rangle\langle 00|\otimes| 00\rangle\langle 00|$ are projection operators onto the triplet and singlet subspaces of qubits $a$ and $b$ and $|J M\rangle$ denote the corresponding (pure) two-qubit quantum states with total angular momentum quantum numbers $J$ and magnetic quantum numbers $M$. The states $\left\{|i\rangle_{3} ; i=0,1\right\}$ and $\left\{|j\rangle_{4} ; j=0,1\right\}$ denote orthonormal basis states in the one-qubit Hilbert spaces of qubits three and four, respectively. According to Eqs. (22) and (23) the four Kraus operators 11] $\mathcal{K}_{i j}(i, j=0,1)$ characterize a quantum operation which acts on the two input qubits one and two and which depends on their degree of entanglement $\alpha$. These Kraus operators map two-qubit states into four-qubit states and they fulfill the complete- ness relation

$$
\sum_{i, j=0,1} \mathcal{K}_{i j}^{\dagger} \mathcal{K}_{i j}=\mathbf{1}_{12}
$$

where $\mathbf{1}_{12}$ denotes the unit operator in the Hilbert space of qubits one and two. Thus, Eq.(22) describes a deterministic quantum operation 11, 12, 13 acting on the two input-qubits which are to be copied in an optimal way. In addition, the Kraus representation of Eq. (22) demonstrates that the optimal covariant copying processes considered so far are completely positive quantum maps [1, 13.

Alternatively, the quantum operation of Eq. (22) may also be implemented by an associated unitary transformation $U$ which involves two additional ancilla-qubits. Denoting orthonormal basis states of these additional ancilla-qubits by $\left\{|\alpha\rangle_{5} \otimes|\beta\rangle_{6} ; \alpha, \beta=0,1\right\}$ this unitary transformation $U$ can be choosen so that it fulfills the relation

$$
U|\psi\rangle_{12} \otimes|0\rangle_{3456}=\sum_{i, j=0,1}\left(\frac{K}{2}|\psi\rangle_{12} \otimes|i\rangle_{3} \otimes|j\rangle_{4}\right) \otimes|i\rangle_{5} \otimes|j\rangle_{6}
$$

for example, with $|0\rangle_{3456}=|0\rangle_{3} \otimes|0\rangle_{4} \otimes|0\rangle_{5} \otimes|0\rangle_{6}$. Thereby, the subscripts of the state vectors label the qubits they are referring to and the bracket of Eq.25. indicates that the Kraus operators act on the systemand device-qubits only. Due to the completeness relation (24) the linear transformation of Eq. (25) preserves 
scalar products, i.e. ${ }_{3456}\left\langle 0\left|\otimes_{12}\left\langle\psi\left|U^{\dagger} U\right| \Phi\right\rangle_{12} \otimes\right| 0\right\rangle_{3456}={ }_{12}$ $\langle\psi \mid \Phi\rangle_{12}$. Thus, it can be completed to a unitary transformation acting on the six-qubits constituted by the system, the device-, and the two ancilla-qubits [13]. Accordingly, the optimal covariant copying process of Eq. 22 can be realized also with the help of this unitary transformation $U$ in the following way: In a first step one applies the transformation $U$ to the intial state $|\psi\rangle_{12} \otimes|0\rangle_{3456}$ of the system-, device- and ancilla-qubits, i.e.

$$
U|\psi\rangle_{12} \otimes|0\rangle_{34563456}\left\langle 0\left|\otimes_{12}\left\langle\psi\left|U^{\dagger}=\sum_{i, j, i^{\prime}, j^{\prime}=0,1} \mathcal{K}_{i j}\right| \psi\right\rangle_{12} \otimes\right| i\right\rangle_{5} \otimes|j\rangle_{6}\left\langle j_{6}\left|\dot{j}^{\prime}\right| i_{5}\right| \otimes_{12}\langle\psi| \mathcal{K}_{i^{\prime} j^{\prime}}^{\dagger}
$$

In a second step one measures the ancilla-qubits in the orthogonal basis $\left\{|\alpha\rangle_{5} \otimes|\beta\rangle_{6} ; \alpha, \beta=0,1\right\}$ without selection of the measurement results. This non-selective measurement [12] yields the output state $\rho_{\text {out }}(|\psi\rangle\langle\psi|)$ of Eq.(22).

\section{PROPERTIES OF OUTPUT STATES}

In this section the degree of entanglement and statistical correlations of the output states produced by the optimal covariant copying processes are discussed.

As the process of copying of an arbitrary pure entangled two-qubit state is not perfect the original and the copy will exhibit characteristic entanglement features and statistical correlations. Convenient measures for quantifying these properties are the concurrences [16] and the indices of correlation [17] of the subsystems.

Let us consider a two-qubit state described by a density operator $\rho$. Its concurrence is defined in terms of the decreasing set of eigenvalues, say $\left\{\lambda_{1} \geq \lambda_{2} \geq \lambda_{3} \geq \lambda_{4}\right\}$, of the operator

$$
R=\rho\left(\sigma_{y} \otimes \sigma_{y}\right) \rho^{*}\left(\sigma_{y} \otimes \sigma_{y}\right) .
$$

Thereby,

$$
\sigma_{y}=\left(\begin{array}{rr}
0 & -i \\
i & 0
\end{array}\right)
$$

denotes the Pauli spin operator and the star-symbol $\left({ }^{*}\right)$ denotes complex conjugation. In terms of these eigenvalues the concurrence of the quantum state $\rho$ is defined by the relation [16]

$$
C(\rho)=\max \left\{0, \sqrt{\lambda_{1}}-\sqrt{\lambda_{2}}-\sqrt{\lambda_{3}}-\sqrt{\lambda_{4}}\right\} .
$$

According to this definition the values of the concurrence are confined to the interval $[0,1]$ with $C(\rho)=0$ and $C(\rho)=1$ corresponding to a separable and a maximally entangled two-qubit state.

A convenient measure for quantifying bipartite statistical correlations of a quantum state $\rho$ is its index of correlation $I(\rho)$ [17]. It is defined by the relation

$$
I(\rho)=S\left(\rho_{a}\right)+S\left(\rho_{b}\right)-S(\rho) ;
$$

with $S\left(\rho_{a}\right), S\left(\rho_{b}\right)$, and $S(\rho)$ denoting the von Neumannentropies of subsystems $a, b$ and of the whole system. The corresponding reduced density operators of the subsystems are denoted by $\rho_{j}=\operatorname{Tr}_{i \neq j}(\rho)$ with $j=a, b$. Correspondingly, the index of correlation $I(\rho)$ vanishes for all uncorrelated (factorizable) states and it attains its largest value for maximally entangled pure states (with $\alpha=1 / \sqrt{2}$ ). Let us now investigate entanglement and statistical correlations of the output state $\rho_{\text {out }}\left(\rho_{\text {in }}\right)$ with respect to the first and the second qubit, with respect to the first and third qubit and with respect to qubits one and two on the one hand and qubits three and four on the other hand.

\section{A. Entanglement and statistical correlations of qubits one and two}

Let us consider first of all a two-qubit input state of the form $\rho_{\text {in }}=|\psi\rangle\langle\psi|$ with $|\psi\rangle \in \Omega_{\alpha}$. Both its concurrence as well as its index of correlation are given by

$$
\begin{aligned}
C\left(\rho_{i n}\right) & =2 \mid \alpha \sqrt{1-\alpha^{2}}, \\
I\left(\rho_{i n}\right) & =-2\left\{|\alpha|^{2} \ln |\alpha|^{2}+|\beta|^{2} \ln |\beta|^{2}\right\}
\end{aligned}
$$

with $\beta=\sqrt{1-\alpha^{2}}$. The corresponding reduced density operator $\rho_{\text {out }}^{(1,2)}$ of qubits one and two after an optimal covariant copying process can be determined easily from Eqs.(8), (11), (20), and (21). In particular, its concurrence, for example, is given by

$$
C\left(\rho_{\text {out }}^{(1,2)}\right)=\frac{1}{16} \max \left\{0,(4|\alpha \beta|+1)\left(2 A_{6}+A_{11}\right)-8\right\} .
$$

The corresponding index of correlation can also be evaluated easily. Due to the inherent symmetry of the optimal covariant copying process the reduced density operators of qubits one and two on the one hand and qubits three and four on the other hand are equal. Therefore, all results obtained for the system-qubits one and two are also valid for the device-qubits three and four.

In Fig 2] the concurrence of the quantum states of qubits one and two before and after the optimal covariant copying process are depicted. The concurrence of 


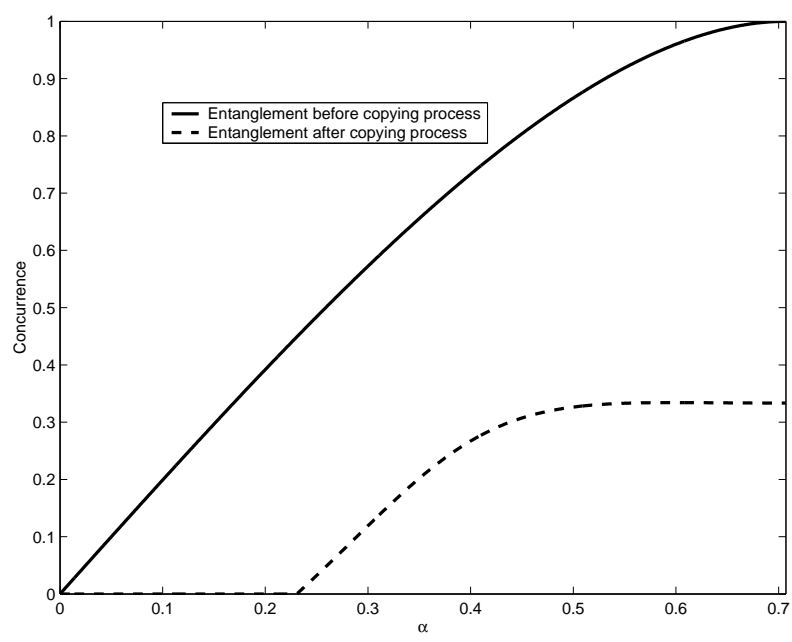

FIG. 2: The dependence of the concurrence of the quantum states of qubits one and two before (solid line) and after (dashed line) the optimal covariant copying process on the degree of entanglement $\alpha . \alpha=0$ and $\alpha=1 / \sqrt{2}$ correspond to the limits of a separable and a maximally entangled pure two-qubit input state.

the pure input state increases smoothly from its minimum value zero at $\alpha=0$ to its maximum value of unity at $\alpha=1 / \sqrt{2}$. The corresponding values of the output states with respect to qubits one and two exhibit a rather different behavior. First of all, it is apparent that a minimum degree of entanglement $\alpha_{\min }=0.231$ of the pure input state $\rho_{\text {in }}$ is required in order to achieve also an entanglement between qubits one and two in the resulting output state. Secondly, the concurrence of the output state saturates at a rather moderate value around 0.3 at which it becomes almost independent of the value of $\alpha$. Thirdly, the maximum entanglement between qubits one and two is not achieved exactly for maximally entangled initial states with $\alpha=1 / \sqrt{2}$ but for values slightly below. However, this difference is very small.

The corresponding indices of correlations and their dependence on the degree of entanglement of input and output states are depicted in Fig 3 In contrast to the concurrence the index of correlation of the output state increases smoothly with increasing values of $\alpha$ and reaches its maximum exactly at $\alpha=1 / \sqrt{2}$. Similar to the case of the concurrence there is a considerable drop of the index of correlation of the copied pair in comparison with its original.

\section{B. Correlation of the first and third qubit}

In view of the structure of the input state $\rho_{0}$ of Eq.(15) the entanglement and statistical correlation between qubits one and three vanish. The concurrence of the reduced density operator of the output state of the optimal covariant copying process with respect to these

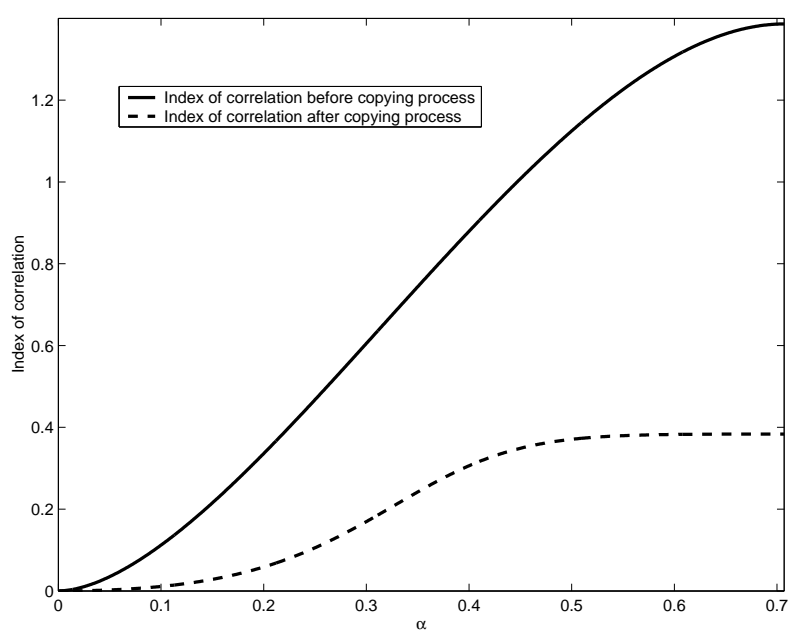

FIG. 3: Indices of correlations of input and output states with respect to qubits one and two and their dependence on the degree of entanglement $\alpha$ of the pure input state. As in the case of concurrence the correlations saturate at a rather moderate level.

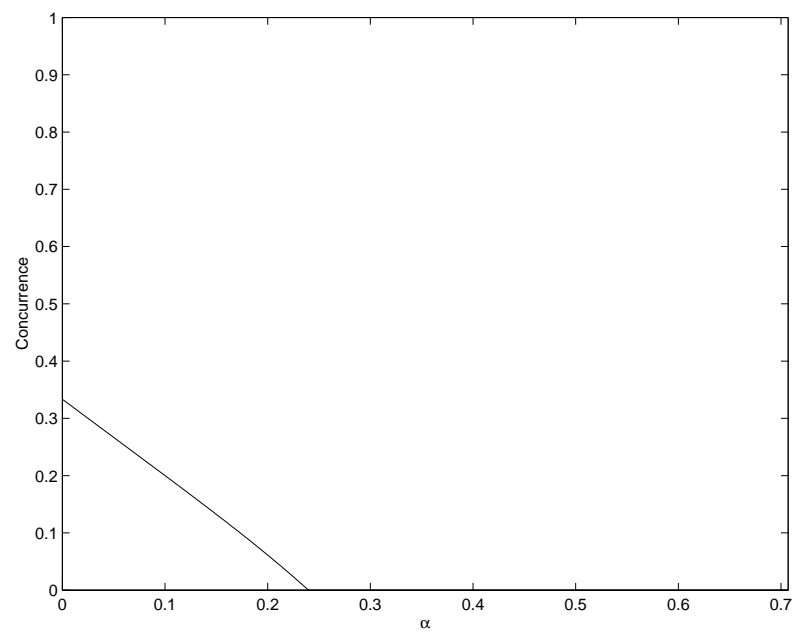

FIG. 4: Concurrence of the output state of an optimal covariant copying process with respect to qubits one and three and its dependence on the degree of entanglement $\alpha$ of the pure two-qubit input state. For $\alpha \geq 0.241$ the concurrence vanishes.

qubits is given by

$$
C_{13}^{(\text {out })}=\frac{1}{4} \max \left\{0,\left|-4+3 A_{6}\right|-3 A_{6} \alpha \sqrt{1-\alpha^{2}}\right\} .
$$

This concurrence and the corresponding index of correlation of the output state and their dependence on the degree of entanglement $\alpha$ of the input state are depicted in Figs 4 and 5 Characteristically, the concurrence decreases linearly from its maximum value at $\alpha=0$ until it vanishes for $\alpha \geq 0.241$. Contrary to the concurrence the index of correlation depends smoothly on the degree of entanglement $\alpha$ of the pure input state. Furthermore, it decreases up to the value $\alpha \approx 0.421$ where it assumes 


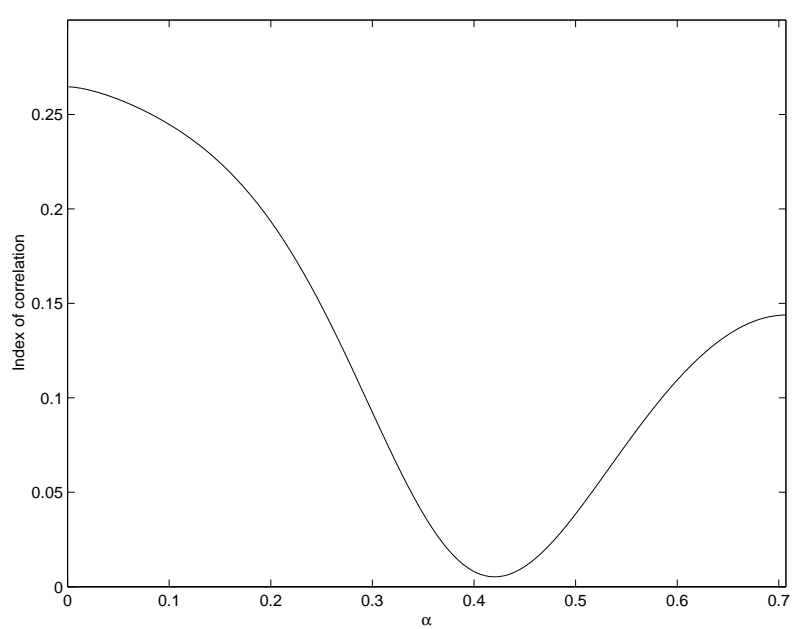

FIG. 5: Index of correlation of the output state of an optimal covariant copying process with respect to qubits one and three and its dependence on the degree of entanglement $\alpha$ of the pure two-qubit input state.

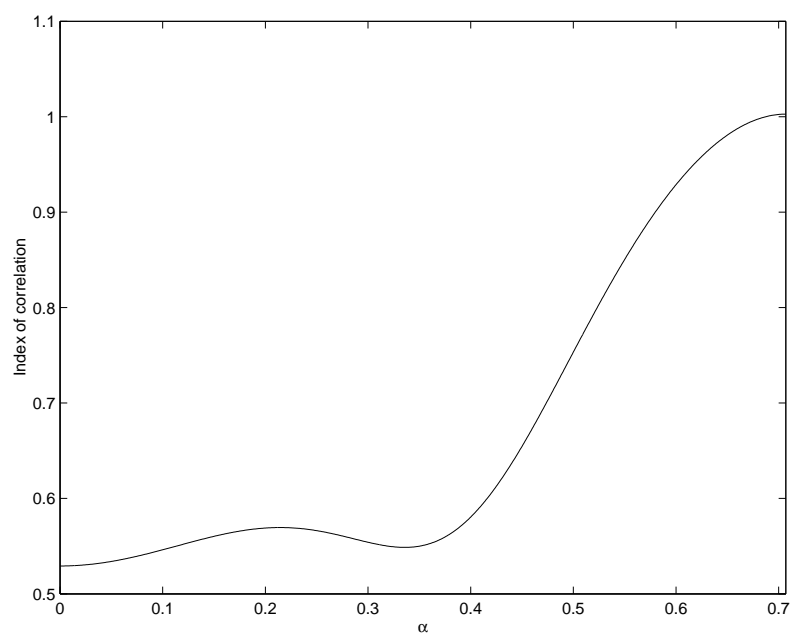

FIG. 6: Index of correlation of the output state of an optimal covariant copying process with respect to qubits one and two and qubits three and four and its dependence on the degree of entanglement $\alpha$ of the pure two-qubit input state.

a minimum. For degrees of entanglement $\alpha \geq 0.421$ it increases monotonically and reaches a local maximum at $\alpha=1 / \sqrt{2}$ which corresponds to a maximally entangled pure input state.

\section{Correlation between two copies}

Finally, let us discuss the statistical correlations of the output state with respect to qubits one and two on the one hand and qubits three and four on the other hand. Its dependence on the degree of entanglement $\alpha$ of the pure two-qubit input state is depicted in Fig[6] Characteristically, one notices two maxima and two minima.

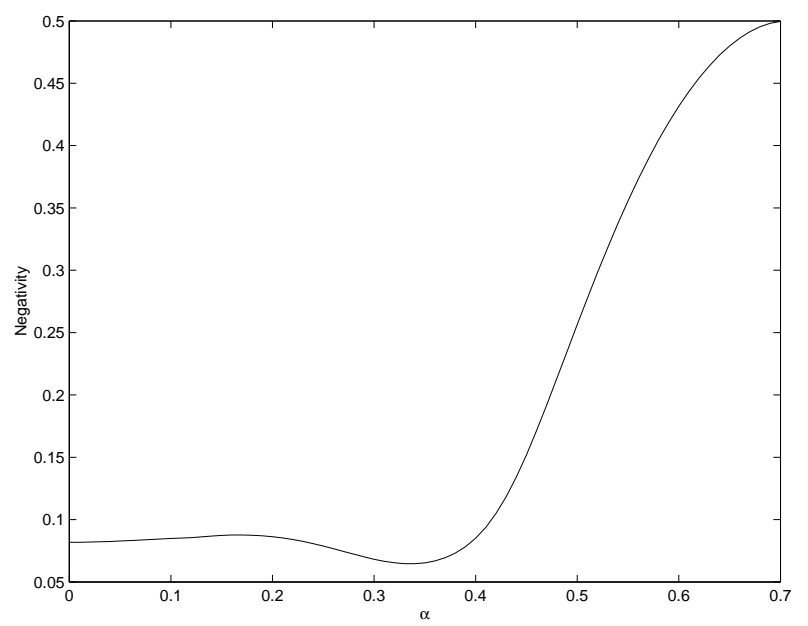

FIG. 7: Negativity of the output state of an optimal covariant copying process and its dependence on the degree of entanglement $\alpha$ of the pure two-qubit input state. The negativity is for all values of $\alpha$ positive a hence the outputs states are always entangled.

The local minimum at $\alpha=\sqrt{1 / 2-\sqrt{15} / 10}$ corresponds to the optimal covariant copying process with the smallest fidelity of the output copies (compare with Fig 1). The global maximum corresponds to the copying of maximally entangled pure input states with $\alpha=1 / \sqrt{2}$.

To conclude our discussion of entanglement and correlations let us consider the negativity of the output state $\rho_{\text {out }}\left(\rho_{\text {in }}\right)$. This quantity allows to decide whether a quantum state contains free entanglement or not. The definition of the negativity of a quantum state starts from the observation, that the partial transpose of a separable state always yields a positive density operator. The negativity is defined by the sum of absolute values of the negative eigenvalues of the partially transposed density operator [18]. In Fig 7 the negativity of the output state is depicted. Thereby, the four-qubit output state is considered as a bipartite state with respect to the systemqubits one and two and the device-qubits three and four. The dependence of this negativity on the degree of entanglement $\alpha$ of the input states resembles the corresponding dependence of the correlation function presented in Fig 6 Fig 17 illustrates several interesting features: Firstly, the output state is entangled for all values of $\alpha$. Secondly, the global minimum of the negativity coincides with the point of worst copying, i.e. with $\alpha=\sqrt{1 / 2-\sqrt{15} / 10}$. The maximum at the point $\alpha=1 / \sqrt{2}$ indicates that the copying of maximally entangled states results in a maximally entangled output state.

\section{CONCLUSION}

We investigated the copying of pure entangled twoqubit states of a given degree of entanglement. Optimiz- 
ing these processes with respect to two separable copies we identified the optimal covariant and completely positive copying processes for all possible degrees of entanglement. It was demonstrated that the fidelity of the resulting output states with respect to separable copies varies between the values of 0.4 and 0.5 . In particular, this latter value characterizes the optimal copying of maximally entangled two-qubit states. In the special case of factorizable input states we obtain the already known value of 4/9. We investigated correlation properties and the entanglement of the resulting output states. We want to point out that the presented approach which is based on an analysis of the irreducible tensor components of the input state may be generalized also to more complex situations, such as the copying of $N$ entangled pairs to $M$ pairs. Work along these lines is in progress.

\section{Acknowledgments}

Financial support by GAČR 202/04/2101, VZ MŠMT 210000018 of the Czech Republic, by the Alexander von Humboldt foundation and by the Deutsche Forschungsgemeinschaft (SPP QUIV) is gratefully acknowledged.

\section{APPENDIX A: OPTIMAL COPYING PROCESSES AND COVARIANT QUANTUM MAPS}

The proof that any optimal quantum copying process $\widehat{T_{\alpha}}$ of the form (2) can be represented by a corresponding covariant quantum map of the form of Eq. (4) with the same fidelity is similar to the proof given by Werner in the context of optimal cloning of arbitrary $d$-dimensional quantum states [4]. The only major difference concerns the group operations which connect all possible pure input states. In our case this group involves all unitary transformations of the form $U_{1} \otimes U_{2}$ with $U_{j} \in S U_{2}$. For the sake of completeness we briefly outline the main steps involved in this proof.

Let us start from the definition of the optimal fidelity of our copying process which is given by

$\widehat{\mathcal{L}_{\alpha}}=\sup _{T_{\alpha}} \mathcal{L}\left(T_{\alpha}\right)=\sup _{T_{\alpha}}\{\inf _{|\psi\rangle \in \Omega_{\alpha}} \underbrace{\left\langle\psi\left|\otimes\left\langle\psi\left|\rho_{\text {out }}\right| \psi\right\rangle \otimes\right| \psi\right\rangle}_{Z_{\psi}}\}$.

The functions $Z_{\psi}$ are continuous. Largest lower bounds (infima) of a set of continuous functions yield upper semicontinuous functions. In a finite dimensional Hilbert space the set of admissible quantum operations $T_{\alpha}$ is closed, and bounded. Therefore it constitutes a compact set. However, an upper semicontinuous function on a compact set must acquire its maximum. Let us denote this maximum $\widehat{T_{\alpha}}$. Thus

$$
\widehat{\mathcal{L}_{\alpha}}=\sup _{T_{\alpha}} \mathcal{L}\left(T_{\alpha}\right)=\mathcal{L}\left(\widehat{T}_{\alpha}\right) .
$$

For an arbitrary admissible quantum copying map $T_{\alpha}$ its average $\tilde{T}_{\alpha}$ over all group operations is defined by

$$
\tilde{T}_{\alpha}(\rho)=\int d U_{1} d U_{2} \mathcal{U}^{* \otimes 2} T_{\alpha}\left(\mathcal{U} \rho \mathcal{U}^{*}\right) \mathcal{U}^{\otimes 2}
$$

with $\mathcal{U}=U_{1} \otimes U_{2}$. Thereby, $d U_{1} d U_{2}$ denotes the normalized left invariant Haar measure of the group $S U(2) \times S U(2)$. The map $\tilde{T}_{\alpha}$ is again an admissible copying map, which fulfills the covariance condition (4). If $\tilde{T}_{\alpha}$ denotes the average of the optimal copying process $\widehat{T}_{\alpha}$, then for every pure state $|\psi\rangle \in \Omega_{\alpha}$ we find

$$
\left\langle\psi\left|\otimes\left\langle\psi\left|\tilde{T}_{\alpha}(|\psi\rangle\langle\psi|)\right| \psi\right\rangle \otimes\right| \psi\right\rangle=\int d U_{1} d U_{2}\left\langle\psi_{\mathcal{U}}\left|\otimes\left\langle\psi_{\mathcal{U}}\left|\tilde{T}_{\alpha}\left(\left|\psi_{\mathcal{U}}\right\rangle\left\langle\psi_{\mathcal{U}}\right|\right)\right| \psi_{\mathcal{U}}\right\rangle \otimes\right| \psi_{\mathcal{U}}\right\rangle \geq \int d U_{1} d U_{2} \mathcal{L}\left(\widehat{T}_{\alpha}\right)=\widehat{\mathcal{L}}_{\alpha}(\mathrm{A} 4)
$$

with $\left|\psi_{\mathcal{U}}\right\rangle=\mathcal{U}|\psi\rangle$. Because the lefthand side of the inequality is independent of $|\psi\rangle \in \Omega_{\alpha}$, it is also valid for $\mathcal{L}\left(\tilde{T}_{\alpha}\right)$, i.e.

$$
\mathcal{L}\left(\tilde{T}_{\alpha}\right) \geq \widehat{\mathcal{L}}_{\alpha}
$$

But from the definition of $\widehat{\mathcal{L}}_{\alpha}$ we know that $\mathcal{L}\left(\tilde{T}_{\alpha}\right) \leq \widehat{\mathcal{L}}_{\alpha}$. Thus, we conclude that

$$
\mathcal{L}\left(\tilde{T}_{\alpha}\right)=\widehat{\mathcal{L}}_{\alpha}
$$

and we can restrict our search for optimal copying processes to covariant ones.

\section{APPENDIX B: IRREDUCIBLE TENSOR OPERATORS}

A set of irreducible tensor operators $T^{(a, b)}\left(J_{1} J_{2}\right)_{K Q}$ for two quantum systems $a$ and $b$ with respect to the rotation group is defined by [14, 15] 


$$
T^{(a, b)}\left(J_{a}, J_{b}\right)_{K Q}=\sum_{M_{a} M_{b}}(-1)^{J_{a}-M_{a}} \sqrt{2 K+1}\left(\begin{array}{ccc}
J_{a} & J_{b} & K \\
M_{a} & -M_{b} & -Q
\end{array}\right)\left|J_{a} M_{a}\right\rangle \otimes\left\langle J_{b} M_{b}\right|
$$

with the ket (bra) $|J M\rangle(\langle J M|)$ denoting eigenstates of the total angular momentum operator of both quantum systems. Thereby the total angular momentum quantum number and the magnetic quantum number are denoted by $J$ and $M$. In Eq.(B1) we have introduced the $3 \mathrm{j}$ symbol whose orthogonality and completeness relations imply the corresponding relations

$$
\operatorname{Tr}\left[T^{(a, b)}\left(J_{a}, J_{b}\right)_{K Q} T^{(a, b)}\left(J_{a}^{\prime}, J_{b}^{\prime}\right)_{K^{\prime} Q^{\prime}}^{\dagger}\right]=\delta_{J_{a} J_{a}^{\prime}} \delta_{J_{b} J_{b}^{\prime}} \delta_{K K^{\prime}} \delta_{Q Q^{\prime}}
$$

Thereby, Tr denotes the trace over the Hilbert spaces of quantum systems $a$ and $b$. The irreducible tensor operators of Eq. B1 are special cases of complete orthogonal sets of operators with simple transformation properties with respect to a particular group. In the case of Eq. (B1) it is the group of rotations of the quantum systems $a$ and $b$ and the simple transformation property is given by

$$
\mathcal{U} T^{(a, b)}\left(J_{a} J_{b}\right)_{K Q} \mathcal{U}^{\dagger}=\sum_{q} T^{(a, b)}\left(J_{a} J_{b}\right)_{K q} D(U)_{q Q}^{(K)} .
$$

with $\mathcal{U}=U \otimes U$.

As the tensor operators of Eq. (B1) form a complete set any operator $\rho$ in the Hilbert space of particles $a$ and $b$ can be decomposed according to

$$
\rho=\sum_{J_{a} J_{b} K Q}\left\langle T^{(a, b)}\left(J_{a} J_{b}\right)_{K Q}^{\dagger}\right\rangle T^{(a, b)}\left(J_{a} J_{b}\right)_{K Q}
$$

with

$$
\begin{aligned}
\left\langle T^{(a, b)}\left(J_{a} J_{b}\right)_{K Q}^{\dagger}\right\rangle & =\operatorname{Tr}\left\{\rho T^{(a, b)}\left(J_{a} J_{b}\right)_{K Q}^{\dagger}\right\}, \quad(\mathrm{B} 5 \\
T^{(a, b)}\left(J_{a} J_{b}\right)_{K Q}^{\dagger} & =(-1)^{J_{a}-J_{b}+Q} T^{(a, b)}\left(J_{b} J_{a}\right)_{K-Q} .
\end{aligned}
$$

With the help of these relations and the condition $U_{1} \otimes$ $U_{2} \rho_{r e f} U_{1}^{\dagger} \otimes U_{2}^{\dagger}=\rho_{\text {ref }}$ which has to be fulfilled by any covariant quantum process it is straightforward to proof the general form of the output state of Eq. (8).

\section{APPENDIX C: POSITIVITY CONSTRAINTS}

We start from the most general form of the output state of the linear and covariant quantum map defined by Eqs. (8) and (11). Due to the covariance condition (4) this output state can be decomposed into a direct sum of density operators according to

with

$$
\rho_{\text {out }}\left(\rho_{\text {in }}\right)=M_{1} \oplus M_{2} \oplus M_{3} \oplus M_{4} \oplus M_{5}
$$

$$
\begin{aligned}
M_{1}= & {\left[\left(2 \alpha^{2}-1\right)\left(A_{2}+A_{5}\right)+A_{1}+A_{6}\right]|11 ; 11\rangle\left\langle 11 ; 11\left|+A_{6}\right| 10 ; 10\right\rangle\left\langle 10 ; 10\left|+A_{8}\right| 10 ; 00\right\rangle\left\langle 10 ; 00\left|+A_{16}\right| 00 ; 00\right\rangle\langle 00 ; 00|+} \\
& A_{14}|00 ; 10\rangle\left\langle 00 ; 10\left|+\left[\left(1-2 \alpha^{2}\right)\left(A_{2}+A_{5}\right)+A_{1}+A_{6}\right]\right| 00 ; 1-1\right\rangle\langle 00 ; 1-1|+2 \alpha \sqrt{1-\alpha^{2}}\left[A_{1}|11 ; 11\rangle\langle 10 ; 10|+\right. \\
& \left.A_{1}|10 ; 10\rangle\langle 11 ; 11|\right]-2 \alpha \sqrt{1-\alpha^{2}}\left[A_{3}|11 ; 11\rangle\left\langle 10 ; 00\left|+A_{3}^{*}\right| 10 ; 00\right\rangle\langle 11 ; 11|\right]+ \\
& 2 \alpha \sqrt{1-\alpha^{2}}\left[A_{11}\left[|11 ; 11\rangle\left\langle 00 ; 00\left|+A_{11}^{*}\right| 00 ; 00\right\rangle\langle 11 ; 11|\right]-2 \alpha \sqrt{1-\alpha^{2}}\left[A_{9}\left[|11 ; 11\rangle\left\langle 00 ; 10\left|+A_{9}^{*}\right| 00 ; 10\right\rangle\langle 11 ; 11|\right]+\right.\right. \\
& \left(2 \alpha^{2}-1\right)\left[A_{7}|10 ; 10\rangle\left\langle 10 ; 00\left|+A_{7}^{*}\right| 10 ; 00\right\rangle\langle 10 ; 10|\right]+\left[A_{11}|10 ; 10\rangle\left\langle 00 ; 00\left|+A_{11}^{*}\right| 00 ; 00\right\rangle\langle 10 ; 10|\right]+
\end{aligned}
$$




$$
\begin{aligned}
& \left(2 \alpha^{2}-1\right)\left[A_{10}|10 ; 10\rangle\left\langle 00 ; 10\left|+A_{10}^{*}\right| 00 ; 10\right\rangle\langle 10 ; 10|\right]+2 \alpha \sqrt{1-\alpha^{2}}\left[A_{1}|10 ; 10\rangle\left\langle 00 ; 1-1\left|+A_{1}\right| 00 ; 1-1\right\rangle\langle 10 ; 10|\right]+ \\
& \left(2 \alpha^{2}-1\right)\left[A_{12}|10 ; 00\rangle\left\langle 00 ; 00\left|+A_{12}^{*}\right| 00 ; 00\right\rangle\langle 10 ; 00|\right]+\left[A_{17}|10 ; 00\rangle\left\langle 00 ; 10\left|+A_{17}^{*}\right| 00 ; 10\right\rangle\langle 10 ; 00|\right]+ \\
& 2 \alpha \sqrt{1-\alpha^{2}}\left[A_{3}^{*}|10 ; 00\rangle\left\langle 00 ; 1-1\left|+A_{3}\right| 00 ; 1-1\right\rangle\langle 10 ; 00|\right]+\left(2 \alpha^{2}-1\right)\left[A_{15}^{*}|00 ; 00\rangle\left\langle 00 ; 10\left|+A_{15}\right| 00 ; 10\right\rangle\langle 00 ; 00|\right]+ \\
& 2 \alpha \sqrt{1-\alpha^{2}}\left[A_{11}^{*}|00 ; 00\rangle\left\langle 00 ; 1-1\left|+A_{11}\right| 00 ; 1-1\right\rangle\langle 00 ; 00|\right]+ \\
& 2 \alpha \sqrt{1-\alpha^{2}}\left[A_{9}^{*}|00 ; 10\rangle\left\langle 00 ; 1-1\left|+A_{9}\right| 00 ; 1-1\right\rangle\langle 00 ; 10|\right], \\
M_{2}= & {\left.\left.\left[\left(2 \alpha^{2}-1\right) A_{4}+A_{8}\right)\right]|11 ; 00\rangle\langle 11 ; 00|+\left[-\left(2 \alpha^{2}-1\right) A_{5}+A_{6}\right)\right]|10 ; 1-1\rangle\langle 10 ; 1-1|+} \\
& {\left.\left.\left[-\left(2 \alpha^{2}-1\right) A_{13}+A_{14}\right)\right]|00 ; 1-1\rangle\langle 00 ; 1-1|+\left[\left(2 \alpha^{2}-1\right) A_{2}+A_{6}\right)\right]|11 ; 10\rangle\langle 11 ; 10|+} \\
& 2 \alpha \sqrt{1-\alpha^{2}}\left[A_{3}^{*}|11 ; 00\rangle\left\langle 10 ; 1-1\left|+A_{3}\right| 10 ; 1-1\right\rangle\langle 11 ; 00|\right]- \\
& \left.2 \alpha \sqrt{1-\alpha^{2}}\left[A_{17}|11 ; 00\rangle\left\langle 00 ; 1-1\left|+A_{17}^{*}\right| 00 ; 1-1\right\rangle\langle 11 ; 00|\right]+\left[\left(2 \alpha^{2}-1\right) A_{7}^{*}+A_{3}^{*}\right)\right]|11 ; 00\rangle\langle 11 ; 10|+ \\
& {\left.\left[\left(2 \alpha^{2}-1\right) A_{7}+A_{3}\right)\right]|11 ; 10\rangle\left\langle 11 ; 00\left|+\left[\left(2 \alpha^{2}-1\right) A_{10}-A_{9}\right]\right| 10 ; 1-1\right\rangle\langle 00 ; 1-1|+} \\
& {\left[\left(2 \alpha^{2}-1\right) A_{10}^{*}-A_{9}^{*}\right]|00 ; 1-1\rangle\langle 10 ; 1-1|+2 \alpha \sqrt{1-\alpha^{2}}\left[A_{1}|10 ; 1-1\rangle\left\langle 11 ; 10\left|+A_{1}\right| 11 ; 10\right\rangle\langle 10 ; 1-1|\right]-} \\
& 2 \alpha \sqrt{1-\alpha^{2}}\left[A_{9}^{*}|00 ; 1-1\rangle\left\langle 11 ; 10\left|+A_{9}\right| 11 ; 10\right\rangle\langle 00 ; 1-1|\right], \\
M_{3}= & {\left.\left.\left[-\left(2 \alpha^{2}-1\right) A_{4}+A_{8}\right)\right]|1-1 ; 00\rangle\langle 1-1 ; 00|+\left[\left(2 \alpha^{2}-1\right) A_{5}+A_{6}\right)\right]|10 ; 11\rangle\langle 10 ; 11|+} \\
& {\left.\left.\left[\left(2 \alpha^{2}-1\right) A_{13}+A_{14}\right)\right]|00 ; 11\rangle\langle 00 ; 11|+\left[-\left(2 \alpha^{2}-1\right) A_{2}+A_{6}\right)\right]|1-1 ; 10\rangle\langle 1-1 ; 10|-} \\
& 2 \alpha \sqrt{1-\alpha^{2}}\left[A_{3}^{*}|1-1 ; 00\rangle\left\langle 10 ; 11\left|+A_{3}\right| 10 ; 11\right\rangle\langle 1-1 ; 00|\right]- \\
& 2 \alpha \sqrt{1-\alpha^{2}}\left[A_{17}|1-1 ; 00\rangle\left\langle 00 ; 11\left|+A_{17}^{*}\right| 00 ; 11\right\rangle\langle 1-1 ; 00|\right]+\left[\left(2 \alpha^{2}-1\right) A_{7}^{*}-A_{3}^{*}\right]|1-1 ; 00\rangle\langle 1-1 ; 10|+ \\
& {\left[\left(2 \alpha^{2}-1\right) A_{7}-A_{3}\right]|1-1 ; 10\rangle\langle 1-1 ; 00|+} \\
& {\left[\left(2 \alpha^{2}-1\right) A_{10}+A_{9}\right]|10 ; 11\rangle\left\langle 00 ; 11\left|+\left[\left(2 \alpha^{2}-1\right) A_{10}^{*}+A_{9}^{*}\right]\right| 00 ; 11\right\rangle\langle 10 ; 11|+} \\
& 2 \alpha \sqrt{1-\alpha^{2}}\left[A_{1}|10 ; 11\rangle\left\langle 1-1 ; 10\left|+A_{1}\right| 1-1 ; 10\right\rangle\langle 10 ; 11|\right]+ \\
& 2 \alpha \sqrt{1-\alpha^{2}}\left[A_{9}^{*}|00 ; 11\rangle\left\langle 1-1 ; 10\left|+A_{9}\right| 1-1 ; 10\right\rangle\langle 00 ; 11|\right], \\
M_{4}= & {\left[\left(2 \alpha^{2}-1\right)\left(A_{2}-A_{5}\right)-A_{1}+A_{6}\right]|11 ; 1-1\rangle\langle 11 ; 1-1|, } \\
M_{5}= & {\left[\left(2 \alpha^{2}-1\right)\left(-A_{2}+A_{5}\right)-A_{1}+A_{6}\right]|1-1 ; 11\rangle\langle 1-1 ; 11| . } \\
&
\end{aligned}
$$

Thereby, the basis states $\left|J M ; J^{\prime} M^{\prime}\right\rangle$ involve eigenstates of the total angular momenta of qubits one and three on the one hand and qubits two and four on the other hand, i.e. $\left|J M ; J^{\prime} M^{\prime}\right\rangle=|J M\rangle_{(1,3)} \otimes\left|J^{\prime} M^{\prime}\right\rangle_{(2,4)}$ with $(J, M)$ and $\left(J^{\prime}, M^{\prime}\right)$ denoting the relevant total angular momentum and magnetic quantum numbers.

The non-negativity of the output state (C2) necessarily implies that all diagonal matrix elements have to be non-negative. The resulting constraints give rise to the inequalities (13). Furthermore, for appropriately chosen pure states $|\chi\rangle$ the relation $\left\langle\chi\left|\rho_{\text {out }}\left(\rho_{\text {in }}\right)\right| \chi\right\rangle \geq 0$ yields the inequalities (14), i.e.

$$
\begin{aligned}
|\chi\rangle= & a|10 ; 10\rangle+b|00 ; 00\rangle \rightarrow\left|A_{11}\right|^{2} \leq A_{16} A_{6}, \\
|\chi\rangle= & a|10 ; 00\rangle+b|00 ; 10\rangle \rightarrow\left|A_{17}\right|^{2} \leq A_{14} A_{8}, \\
|\chi\rangle= & a|11 ; 11\rangle+b|00 ; 1-1\rangle+c|10 ; 10\rangle \\
\rightarrow & A_{6}\left|\left(2 \alpha^{2}-1\right)\left(A_{2}+A_{5}\right)\right|^{2} \leq\left(A_{1}+A_{6}\right)^{2} A_{6}- \\
& 8 \alpha^{2}\left(1-\alpha^{2}\right) A_{1}^{2}\left(A_{1}+A_{6}\right)
\end{aligned}
$$

with $a, b$ and $c$ denoting arbitrary complex-valued coefficients.
[1] W. K. Wooters and W. H. Zurek, Nature (London) 299, 802 (1982).

[2] V. Bužek and M. Hillery, Phys. Rev. A 54, 1844 (1996); V. Bužek and M. Hillery, Phys. Rev. Lett. 81, 5003 (1998).

[3] N. Gisin and S. Massar, Phys. Rev. Lett. 79, 2153 (1997).

[4] R. Werner, Phys. Rev. A 58, 1827 (1998).

[5] C.-S. Niu and R. B. Griffiths, Phys. Rev. A 58, 4377 (1998)

[6] N. J. Cerf, Phys. Rev. Lett. 84, 4497 (2000).

[7] N. J. Cerf, A. Ipe, and X. Rottenberg, Phys. Rev. Lett.
85, 1754 (2000); N. J. Cerf and S. Iblisdir, Phys. Rev A 62, 040301 (2000); S. L. Braunstein, N. J. Cerf, S. Iblisdir, P. van Loock, and S. Massar, Phys. Rev. Lett. 86, 4438 (2001); N. J. Cerf and S. Iblisdur, Phys. Rev. Lett. 87, 247903 (2001).

[8] S. L. Braunstein, V. Bužek, and M. Hillery, Phys. Rev. A 63, 052313 (2001).

[9] J. Fiurášek, S. Iblisdir, S. Massar, and N. J. Cerf, Phys. Rev. A 65, 040302(R) (2002).

[10] L.-P. Lamoureux, P. Navez, J. Fiurášek, and N. J. Cerf, Phys. Rev. A 69, 040301-1 (2004). 
[11] K. Kraus, States, Effects, and Operations: Fundamental Notions of Quantum Theory, Lecture Notes in Physics 190 (Springer, Berlin, 1983).

[12] A. Peres, Quantum Theory: Concepts and Methods (Kluwer Academic Pub., Dordrecht, 1995).

[13] M. A. Nielsen and I. L. Chuang, Quantum Computation and Quantum Information (Cambridge UP, Cambridge, 2000).

[14] K. Blum, Density Matrix Theory and Applications (Plenum Press, New York, 1981).

[15] L. C. Biedenharn and J. D. Louck, Angular Momentum in
Quantum Physics (Addison-Wesley Publishing Company, Massachusetts, 1981).

[16] S. Hill, W.K. Wootters, Phys. Rev. Lett. 78, 5022 (1997); W. K. Wooters, Phys. Rev. Lett. 80, 2245 (1998); V. Coffman, J. Kundu, And W. K. Wootters, Phys. Rev. A 61, 052306 (2000).

[17] S. M. Barnett, S. J. D. Phoenix, Phys. Rev. A 40, 2404 (1989).

[18] G. Vidal and R. F. Werner, Phys. Rev.A 65, 032314 (2002). 